background - then it must be acknowledged that in strict logic a system has all the orders of which it is capable, and that which is most familiar, or most immediately perceived, may not be the order. It was Kant's problem as to what justifies us in seeing nature as a whole that assumes the form of a logical system and its solution requires theoretical input concerning causality and not mere assessment of pattern. Cladistics as a formal system is a sterile enterprise; it becomes alive only when it deals in terms of generation and transformation, either as a result of human agency (for example the study of languages or of historical texts) or as a result of processes of nature (evolution). It follows that I find the authors definitions of cladograms as synapomorphy schemes lacking such connotations less than useful, their explicit defence of this position notwithstanding. Others will decide for themselves.

There is also a great deal of set theory in the book, but of a simplistic and misleading sort. If one rejects nominalism, and accepts that sets over which quantification is possible exist, then one cannot arbitrarily deny some and not others. If quantification were not possible over all sorts of (admittedly overlapping) biological sets, then comparative physiology, ecology and so forth would not be possible. Logically, the meiofauna is as valid a set as is Aves. The special sense of existence for the hierarchical nonoverlapping sets representing the higher taxa of biological systematics is meaningful only when causal principles of their origins are involved.

What can one make of discussions of sets that are "synonymous"? Does this mean identicality, proper inclusion? This is r ot always made clear, and some set definitions given in terms of homologous character states may well prove to violate the "vicious circle principle". Concepts of intensional and extensional definition, denotation and connotation, although not explicitly discussed are often confused, and we could have hoped for more profound discussions of "natural" in the sense of "natural classifications", "natural taxa" and "natural kinds". The authors seem unaware that there is a vast philosophical Jitcrature dealing with such concepts and, it seems to me, fail to appreciate that these terms have meanings more precise than those of everyday use. Wc are told that homology does not exist independent of synapomorphy (a clear case of putting the cart before the horse!) any more than a set exists independent of its members, yet most logicians require the null or empty set. I am aware that logicians such as Goodman refer to wholes rather than sets, preferring to do without the null set, but there is no evidence that Nelson and Platnick have taken this particular route.

If I have dwelt on some of the logical and philosophical shortcomings, as I sce them, of Systematics and Biogeography it is because the relevant sections are a very important part of the work. And because they are so lucidly written, despite some lapses into condescension, these sections will have an immediate appeal to those biologists unused to logic and philosophy. Yet they must be read critically, for if it is indeed correct to accept the main conclusions of the book while denying the argument then the philosophy and logic must be re-worked. Nevertheless, I recognize the book as a tour de force of systematic biology; in particular, the synthetic parts such as those linking systematics and biogeography into one grand structure are masterly. And even if in the historical sections scant attention has been made to my heroes, Kant and Goethe - whose notions of natural order are surely relevant to this book - I would still willingly sing with the angels from Faust: "Wer immer strebend sich bemüht, den können wir erlösen".

Ian R. Ball is Professor in Systematic and Geographical Zoology in the University of Amsterdam, The Netherlands.

\title{
Fresh interpretation of the galaxies
}

\section{Barry F. Madore}

A Revised Shapley-Ames Catalog of Bright Galaxies. By Allan Sandage and G.A. Tammann. Pp.157. ISBN 0-87279-652-3. (Carnegie Institution of Washington: 1981.) $\$ 29$.

SEVERAL years ago I walked into the refrigerated plate-storage room of the Dupont telescope at the Las Campanas Observatory in Chile, only to be confronted with a yellow wall of Kodak plate boxes carrying the message "Do not use Reserved for Allan Sandage". These were the plates that were yet to be exposed to the black skies over the Chilean Andes, and which were destined to complete $A$ Revised Shapley-Ames Catalog of Bright Galaxies.

Nearly half a century ago, Harlow Shapley and Adelaide Ames published their now-famous list of some 1,200 galaxies. Although this was only a small subset of the galaxies observed visually by the Herschels (data from which was compiled by Dreyer in the 1888 edition of the New General Catalogue), the ShapleyAmes Catalog bore the distinction of being quantified in a dimension that transcended the NGC and set the stage for subsequent discussions of the spatial distribution and intrinsic properties of galaxies. The original Catalog was, with a fair degree of success, a complete sample of the apparently brightest galaxies to a magnitude limit of about $m_{\mathrm{ou}} \sim 13$. The publication of a revised version by Sandage and Tammann is both a natural extension of this work and a bold expression of new convictions.

A Revised Shapley-Ames Catalog is not simply a listing of positions, magnitudes and classifications for the brightest galaxies, it is a personal statement about galaxies and the type of Universe they inhabit. The new Catalog contains much interpreted data, and in Sandage's own words "This Catalog is going to be controversial". Certainly, most of the controversy will arise from the distances that are hidden away in the tabulated absolute magnitudes of Column 16. And almost as certainly we shall soon see a third edition of the Reference Catalogue of Bright Galaxies by the de Vaucouleurs, who, together with Corwin, aimed at providing a different interpretation of the data. The time and energy needed to follow the tortuous paths of logic encountered in determining the distances to galaxies can only leave lesser mortals following on in awe. So there is and will continue to be a widely accepted need for this type of quantitative speculation.

Still, for those wishing to strike out on their own the revised Catalog will provide a wealth of raw data. Radial velocities for all but a handful of galaxies are now available; a comprehensive reference list to the original data is graciously provided; and excellent photographs illustrate the classification system employed.

As dry as a catalogue might potentially be, there is a sprinkling of humour in the text. For example, in Table 10, describing the codes for the sources of plate material used in the galaxy typing, the Uppsala Schmidt is listed as having been used for a total of zero galaxies. One might wonder how many other telescopes should have also been included here.

Finally, it is of interest, to me in particular, that "at least 98 per cent of the catalog galaxies can be fitted into the revised Hubble system" while "descriptive terms, such as 'pec' (peculiar), 'disrupted', 'tidal', 'ring', 'jet' and 'edge on' have been added" only in several cases. Those who see the perfection and uniformity of nature give a balance to those who study the pathology.

As for controversy, this is surely a sign of health in an active science. $A$ Revised Shapley-Ames Catalog will be a valuable and welcome addition to the continuing search for answers in extragalactic astronomy.

Barry F. Madore is a Professor in the Department of Astronomy at the University of Toronto. 